\title{
Keine Immunsuppressiva bei IgA-Nephritis
}

\author{
Bei einer IgA-Nephritis verlangsamt eine zusätzliche immunsuppressive Therapie nicht \\ die Abnahme der eGFR, sondern geht nur mit unerwünschten Wirkungen einher.
}

_ Zur Basistherapie der IgA-Nephritis gehört eine konsequente Blutdrucksenkung und eine Behandlung der Proteinurie, meist mit ACE-Hemmern oder Angiotensin-Rezeptor-Antagonisten. Nun wurde der Einsatz von Immunsuppressiva in einem offenen, multizentrischen, randomisierten und kontrollierten $\mathrm{Pa}$ rallelgruppenvergleich untersucht.

309 Patienten wurden sechs Monate lang konventionell behandelt. Bei $94 \mathrm{~Pa}$ tienten ging die Proteinurie auf unter 0,75 g/Tag zurück. 162 der verbliebenen Patienten wurden nun entweder weiter supportiv behandelt $(n=80)$ oder erhielten zusätzlich Prednisolon in absteigender Dosierung, Cyclophosphamid und Azathioprin $(n=82)$.
Nach drei Jahren hatten 5\% der supportiv und $17 \%$ der zusätzlich immunsuppressiv behandelten Patienten eine vollständige klinische Remission erreicht. Bei $28 \%$ bzw. 26\% der Patienten ging die geschätzte glomeruläre Filtrationsrate (eGFR) um $>15 \mathrm{ml} / \mathrm{min} / 1,73 \mathrm{~m}^{2}$ zurück. Der jährliche Rückgang unterschied sich nicht signifikant.

Die immunsuppressiv behandelten Patienten erlitten durch diese Behandlung gravierende Nachteile wie schwere Infektionen, gestörte Glukosetoleranz und Gewichtszunahme von mehr als 5 kg im ersten Jahr der Behandlung. Ein Patient aus der immunsuppressiv behandelten Gruppe verstarb sogar an einer Sepsis.
- Rauen Tet al. Intensive support care plus immunosuppression in IgA nephropathy. New Engl J Med. 2015;373:2225-36

\section{KOMMENTAR}

Mit dieser Studie aus Deutschland dürfte die Diskussion um einen möglichen Vorteil einer immunsuppressiven Therapie bei der IgANephritis wohl vorläufig beendet sein. Unabhängig von dem für einen klinischen Wissenschaftler eher deprimierenden Ergebnis gebührt den Autoren und dem New England Journal of Medicine großer Respekt dafür, dass diese Studie überhaupt so prominent publiziert werden konnte. Negativberichte haben es in der Literatur deutlich schwerer, auch wenn die hier eingesetzten Substanzen keine Innovationen der pharmazeutischen Industrie darstellen.

Prof. Dr. med. H. S. FüeßI

\section{Bildgebung enthüllt Bürstenschädel}

Im Rahmen der Abklärung von Kopfschmerzen wurde bei einer 29-jährigen Frau auch ein MRT des Schädels angefertigt, das eine ausgeprägte hypodense Aufweitung der Diploe mit Verdrängung der Tabula externa des Schädelknochens
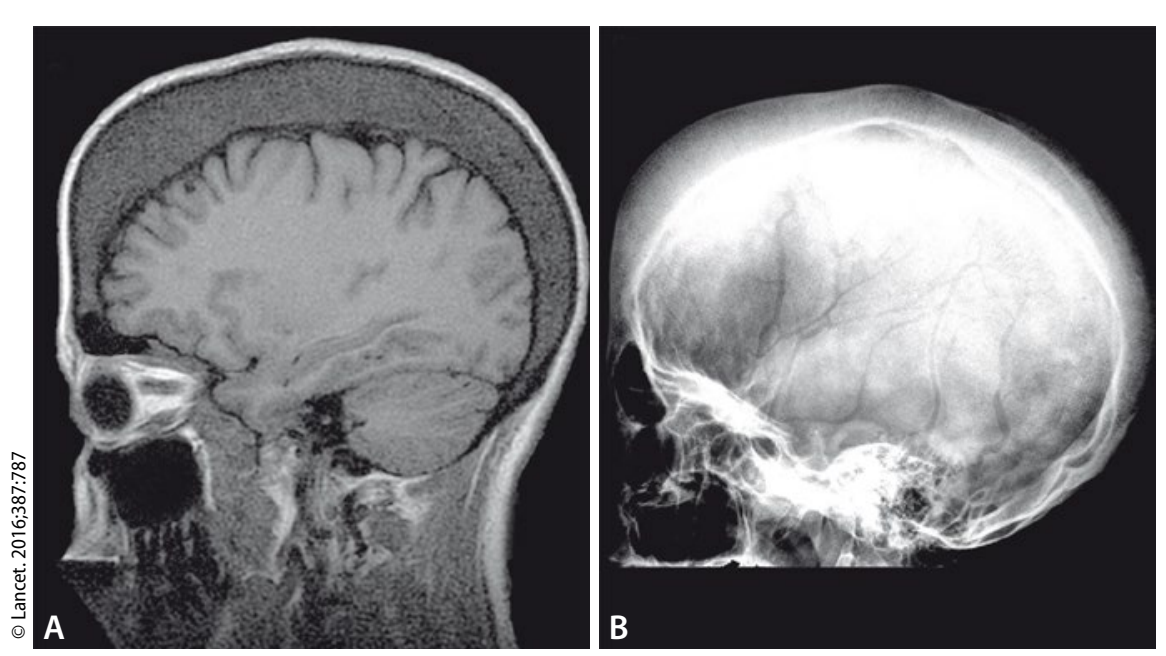

A: Hypodense Aufweitung der Diploe mit Verdrängung der Tabula externa des

Schädelknochens im MRT. B: Darstellung in der Röntgenaufnahme.

zeigte (Abb. A). Wegen einer kongenitalen dyserythropoietischen Anämie vom Typ III hatte die Patientin bis zum 9. Lebensjahr häufige Bluttransfusionen erhalten. In diesem Alter wurde eine Splenektomie durchgeführt, wonach sie bei den jährlichen hämatologischen Kontrollen stabile Hämoglobinwerte um die 9-10 g/dl aufwies. Nachdem das MRT sonst keine Anomalien zeigte, stellte man die Diagnose eines Spannungskopfschmerzes, der gut mit mittelstarken Analgetika und Verhaltenstherapie kontrolliert werden konnte.

Kongenitale dyserythropoietische Anämien gehören zur Gruppe seltener Erkrankungen mit ineffektiver Erythropoese. Die Veränderungen am Schädelknochen entstehen durch eine reaktive Hyperplasie des Knochenmarks mit verstärkter Erythropoese aufgrund der chronischen Anämie. In manchen Fällen stellen sich die Knochentrabekel auf konventionellen Röntgenaufnahmen als strahlenförmig zwischen der Tabula interna und externa verlaufend dar, was zur blumigen Bezeichnung eines Bürsten- oder Haarschädels geführt hat (Abb. B). Besonders eindrucksvolle Befunde sieht man bei Säuglingen und Kleinkindern mit $\beta$-Thalassämie.

Prof. Dr. med. H. S. FüeßI

- Pérez-Jacoiste Asín MA, Ruiz Robles G (mperezja82@ hotmail.com): Skull erythropoiesis in a patient with congenital dyserythropoietic anaemia. Lancet. 2016;387:787 